\title{
AVALIAÇÃO DA PADRONIZAÇÃo INTERNA EM ESPECTROMETRIA DE ABSORÇÃo ATÔMICA
} UTILIZANDO GRÁFICOS DE CORRELAÇÃOO

Paulo R. M. Correia e Pedro V. Oliveira*

Instituto de Química, Universidade de São Paulo, CP 26077, 05513-970 São Paulo-SP

Recebido em 14/6/04; aceito em 14/9/04; publicado na web em 17/2/05

\begin{abstract}
EVALUATION OF INTERNAL STANDARDIZATION IN ATOMIC ABSORPTION SPECTROMETRY USING CORRELATION GRAPHS. The use of an internal standard (IS) in ET AAS can be considered a new trend after the commercial introduction of a simultaneous spectrometer. The evaluation of experimental data to choose the most appropriate IS can be done by comparing correlation graphs. They were used to verify the resemblance among the simultaneous measurements obtained for the analyte(s) and the IS by inductively coupled plasma optical emission spectrometry (ICPOES). The judicious selection of IS by using correlation graphs for determinations by ET AAS can be exploited to improve the precision and accuracy of the analytical results. Therefore, a new approach for studying the use of IS in ET AAS is presented.
\end{abstract}

Keywords: internal standard; simultaneous atomic absorption spectrometry; correlation graphs.

\section{INTRODUÇÃO}

A utilização de padrão interno (PI) em vários procedimentos de análise instrumental tem como objetivo principal melhorar a precisão e a exatidão dos resultados analíticos ${ }^{1,2}$. Para isso, a detecção multielementar, preferencialmente com a aquisição simultânea dos sinais analíticos, é condição essencial e indispensável. Esse fato pode ser considerado como uma das causas responsáveis pela defasagem dos estudos envolvendo a aplicação de PI em espectrometria de absorção atômica (AAS), em comparação com outras técnicas como a espectrometria de emissão óptica com plasma indutivamente acoplado (ICPOES) e a espectrometria de massas com plasma indutivamente acoplado (ICPMS).

Os estudos envolvendo a utilização de PI em ICPOES e ICPMS são numerosos e os benefícios que podem ser obtidos a partir da correção dos resultados analíticos com PI já são conhecidos ${ }^{3-8}$. A avaliação do desempenho do PI pode ser realizada por meio de gráficos de correlação, construídos a partir dos sinais instrumentais normalizados obtidos para o PI, em função dos sinais normalizados obtidos para o analito $^{6}$. Além de facilitar a visualização das respostas analíticas para uma série de medidas consecutivas, os parâmetros obtidos a partir da regressão linear dos gráficos de correlação permitem comparar a eficiência de diferentes elementos utilizados como $\mathrm{PI}^{6}$.

O primeiro estudo propondo a utilização de PI para procedimentos analíticos envolvendo a espectrometria de absorção atômica com atomização eletrotérmica (ET AAS) surgiu somente em 1979. Utilizando um equipamento multicanal, Co foi utilizado como PI na determinação de Fe em amostras de água para reduzir os erros devido às flutuações instrumentais e às interferências químicas provocadas por alguns cátions presentes na matriz.

A introdução comercial de um espectrômetro de absorção atômica simultâneo, durante a década de 90, incorporou o caráter multielementar à ET AAS de maneira definitiva ${ }^{10-12}$. Atualmente, a instrumentação disponível permite detectar até 6 sinais de radiação simultaneamente $^{11}$. Além de melhorar a freqüência analítica, a detecção simultânea tornou possível a utilização de PI em AAS. Após duas décadas da publicação do primeiro estudo ${ }^{9}$, surgiram na litera-

*e-mail: pvolivei@iq.usp.br tura dois trabalhos que exploram $\mathrm{Bi}^{13,14}$ e $\mathrm{Tl}^{13}$ como PI para a determinação de $\mathrm{Pb}$ em amostras clínicas ${ }^{13}$ (urina, sangue e placenta) e vinhos $^{14}$. Nesses trabalhos, um espectrômetro simultâneo foi utilizado para realizar a determinação de $\mathrm{Pb}$ empregando Bi como PI.

A utilização de PI em ET AAS tem sido pouco explorada e as informações existentes na literatura sobre esse tópico estão concentradas em poucos trabalhos científicos ${ }^{9,12-16}$. A precisão dos resultados analíticos tem sido o único parâmetro utilizado para corroborar a adoção de PI nas determinações por ET AAS. A redução do desvio padrão relativo dos resultados analíticos foi o principal argumento que motivou a utilização de Co e Bi como PI para determinar Fe e $\mathrm{Pb}$, respectivamente $\mathrm{e}^{9,12,13}$.

Os gráficos de correlação ainda não foram explorados para avaliar a eficiência da padronização interna em ET AAS. Todavia, essa abordagem se faz necessária para que seja possível obter informações que colaborem com a seleção do PI mais adequado. A comparação do desempenho de vários elementos testados como PI é facilitada quando os gráficos de correlação são utilizados, em adição à comparação do desvio padrão relativo obtido sem e com a padronização interna.

O objetivo do presente trabalho é empregar os gráficos de correlação como uma ferramenta para comparar o desempenho de diferentes elementos testados como PI em ET AAS. Nesse contexto, Ag e Bi serão avaliados como potenciais PI visando a determinação simultânea de $\mathrm{Cd}$ e $\mathrm{Pb}$. A interpretação dos dados experimentais, realizada a partir dos gráficos de correlação, será apresentada considerando-se alguns parâmetros que podem afetar o desempenho do PI, tais como o modificador químico e a temperatura de pirólise.

\section{PARTE EXPERIMENTAL}

\section{Instrumentação}

Um espectrômetro de absorção atômica com atomização eletrotérmica em forno de grafite e detecção simultânea (SIMAA-6000, Perkin-Elmer, Shelton, CT, EUA) foi utilizado no desenvolvimento desse trabalho. O equipamento é dotado de policromador Echelle, tubo de grafite com aquecimento transversal e plataforma integrada recoberta com grafite pirolítico, corretor de radiação de fundo por efeito Zeeman 
longitudinal e amostrador automático (AS-72) para introdução de soluções no interior do atomizador. O espectrômetro utilizado apresenta todos os atributos necessários para operar sob as condições STPF ${ }^{17}$. Os parâmetros instrumentais utilizados, bem como o programa de aquecimento, estão descritos na Tabela 1.

Argônio 99,998\% v/v (Air Liquide, São Paulo, SP) foi utilizado como gás protetor do tubo de grafite e como gás de purga. A vazão de argônio para proteger a superfície externa do tubo de grafite foi fixada pelo fabricante em $200 \mathrm{~mL} \mathrm{~min}^{-1}$. Durante o programa de aquecimento, uma vazão interna de argônio (gás de purga) foi fixada em $250 \mathrm{~mL} \mathrm{~min}^{-1}$ durante as etapas de secagem, pirólise e limpeza, sendo interrompida durante a etapa de atomização. A vazão interna de argônio arrasta os vapores formados durante as etapas de secagem, pirólise e limpeza, bem como protege a superfície interna do tubo de grafite.

\section{Reagentes e soluções}

As soluções analíticas de referência foram preparadas a partir de diluições sucessivas de soluções estoque contendo $1000 \mathrm{mg} \mathrm{L}^{-1}$ (Tritisol, Merck, Darmstadt, Alemanha) de $\mathrm{Ag}\left(\mathrm{AgNO}_{3}\right), \mathrm{Bi}\left(\mathrm{BiCl}_{3}\right)$, $\mathrm{Cd}\left(\mathrm{CdCl}_{2}\right)$ e $\mathrm{Pb}\left[\mathrm{Pb}\left(\mathrm{NO}_{3}\right)_{2}\right]$ : (a) $10 \mu \mathrm{g} \mathrm{L}^{-1} \mathrm{Cd}^{2+}+100 \mu \mathrm{g} \mathrm{L}^{-1} \mathrm{~Pb}^{2+} \mathrm{em}$ $0,1 \% \mathrm{v} / \mathrm{v} \mathrm{HNO}_{3}$; (b) $20 \mu \mathrm{g} \mathrm{L}^{-1} \mathrm{Ag}^{+} \mathrm{em} 0,1 \% \mathrm{v} / \mathrm{v} \mathrm{HNO}_{3}$; (c) $200 \mu \mathrm{g} \mathrm{L}^{-1}$ $\mathrm{Bi}^{3+}$ em $0,1 \%$ v/v $\mathrm{HNO}_{3}$. Uma solução contendo $0,1 \%$ v/v $\mathrm{HNO}_{3}$ na ausência dos elementos de interesse foi preparada e utilizada como branco analítico sempre que necessário.

Os modificadores químicos foram preparados a partir de sais de elevado grau de pureza destinados a esta finalidade: $\mathrm{Pd}\left(\mathrm{NO}_{3}\right)_{2}$, $\mathrm{Mg}\left(\mathrm{NO}_{3}\right)_{2}$ e $\mathrm{NH}_{4} \mathrm{H}_{2} \mathrm{PO}_{4}$ (Suprapur, Merck).

Todas as soluções foram armazenadas em frascos de polipropileno descontaminados (Nalge Company, Rochester, NY, USA).

\section{Procedimento}

Os estudos de repetibilidade foram realizados com soluções analíticas de referência em meio aquoso $\left(0,1 \% \mathrm{v} / \mathrm{v} \mathrm{HNO}_{3}\right)$. Sob condições de aquecimento otimizadas para as etapas de pirólise e atomização (Tabela 1), vinte medidas de absorbância consecutivas foram obtidas para cada solução, contendo os elementos de interesse e o PI em teste. A média, o desvio padrão e o desvio padrão relativo do conjunto de dados obtidos sem e com a correção utilizando PI permitiu realizar uma análise preliminar da eficiência dos elementos selecionados para essa finalidade.

A comparação do comportamento apresentado pelos elementos de interesse e PI foi realizada por meio de gráficos de correlação, conforme indicado na literatura ${ }^{6}$. As 20 medidas de absorbância obtidas para cada elemento durante os testes de repetibilidade foram normalizadas com base no primeiro valor da série. Desta forma, obteve-se os valores normalizados de absorbância para os analitos e para o PI em teste. Posteriormente, um gráfico foi construído a partir dos valores normalizados de absorbância, sendo que no eixo das abscissas são relacionados os dados para o analito e no eixo das ordenadas, os dados para o PI. Desta forma, um gráfico de correlação foi gerado para cada par de elementos composto por um PI e um analito. Por exemplo, nos testes de $\mathrm{Ag}$ como PI para $\mathrm{Cd}$ e $\mathrm{Pb}$ foram gerados dois gráficos de correlação diferentes $(\mathrm{Cd} / \mathrm{Ag}$ e $\mathrm{Pb} / \mathrm{Ag})$, para cada condição experimental utilizada.

A regressão linear dos gráficos de correlação permite avaliar mais detalhadamente o potencial dos elementos testados como PI. Por esse motivo, os parâmetros obtidos a partir da regressão linear desses gráficos (coeficiente linear, coeficiente angular e coeficiente de correlação) foram considerados para comparar o desempenho dos diferentes elementos selecionados como PI.

Todas as medidas experimentais foram baseadas na área integrada do sinal de absorbância.

\section{RESULTADOS E DISCUSSÃO}

\section{A seleção preliminar dos candidatos a PI}

A seleção dos elementos a serem testados como PI visando a determinação simultânea de $\mathrm{Cd}$ e $\mathrm{Pb}$ foi realizada considerando-se os seguintes aspectos: (1) a comparação de parâmetros físicos e químicos relacionados com o processo de atomização desses elemen$\operatorname{tos}^{18}$; (2) a estabilidade térmica obtida para esses elementos na presença dos modificadores químicos usualmente empregados para $\mathrm{Cd}$ e $\mathrm{Pb}^{19,20}$ e (3) as condições de análise recomendadas para procedimentos monoelementares desses elementos ${ }^{20}$. Desta forma, $\mathrm{Ag}$ e $\mathrm{Bi}$ foram selecionados como elementos promissores para serem utilizados como PI para $\mathrm{Cd}$ e $\mathrm{Pb}$.

Tabela 1. Parâmetros instrumentais e programa de aquecimento do forno de grafite

\begin{tabular}{lcccc}
\hline & \multicolumn{2}{c}{ Parâmetros do espectrômetro } & & \\
\hline & $\mathrm{Cd}$ & $\mathrm{Pb}$ & $\mathrm{Bg}$ & \\
\hline$\lambda(\mathrm{nm})$ & 228,8 & 283,3 & 328,1 & 223,1 \\
Fonte de radiação $^{a}$ & $\mathrm{EDL}$ & $\mathrm{EDL}$ & $\mathrm{HCL}$ & $\mathrm{EDL}$ \\
$\mathrm{i}(\mathrm{mA})$ & 230 & 450 & 0,7 & 380 \\
Largura da fenda (nm) & 0,7 & 0,7 & 3 & 0,7 \\
Tempo de integração do sinal (s) & 3 & 3 & 3 \\
\hline
\end{tabular}

Programa de aquecimento

\begin{tabular}{lcccc}
\hline Etapa & Temperatura $\left({ }^{\circ} \mathrm{C}\right)$ & Rampa $(\mathrm{s})$ & Patamar $(\mathrm{s})$ & Vazão de argônio $\left(\mathrm{mL}\right.$ min $\left.{ }^{-1}\right)$ \\
\hline Secagem I & 130 & 10 & 15 & 250 \\
Secagem II & 200 & 5 & 10 & 250 \\
Pirólise & 600 & 5 & 15 & 250 \\
Atomização & $1600-1700$ & 0 & 5 & 0 \\
Limpeza $^{b}$ & 2500 & 1 & 2 & 250 \\
\hline
\end{tabular}

${ }^{a}$ EDL: lâmpada de descarga sem eletrodo ("Electrodeless discharge lamp"), HCL: lâmpada de catodo oco ("Hollow cathode lamp"). ${ }^{b}$ Parâmetro otimizado em função do modificador químico utilizado. Tempo total do programa: $68 \mathrm{~s}$; volume de solução analítica de referência: $10 \mu \mathrm{L}$; volume de modificador químico: $10 \mu \mathrm{L}$; temperatura de injeção da amostra: $100{ }^{\circ} \mathrm{C}$. 


\section{A avaliação dos gráficos de correlação}

Os gráficos de correlação permitem realizar uma avaliação quantitativa do desempenho de diferentes PI para um determinado analito, considerando o grau de similaridade existente entre os sinais instrumentais obtidos para os dois elementos (analito e PI). Os valores normalizados para uma série de medidas consecutivas podem ser representados graficamente e comparados com a situação ideal, isto é, quando se observa uma correlação perfeita entre os sinais do analito e do PI em teste. Os parâmetros obtidos por meio da regressão linear dos pontos do gráfico de correlação são empregados para essa avaliação.

O coeficiente de correlação (r) reflete o grau de similaridade entre as variações dos sinais obtidos para o analito e para o PI. Idealmente, o coeficiente de correlação para esse gráfico deve assumir valor igual a 1 . O coeficiente angular (b) permite avaliar a semelhança entre a amplitude das variações dos sinais obtidos para o analito e para o PI. As variações de intensidade observadas para esses sinais devem ser proporcionais e, caso isso se verifique, o coeficiente angular do gráfico de correlação será igual a 1. Valores diferentes para o coeficiente angular indicam que a correção dos resultados não é eficiente, mostrando que o PI e o analito são afetados de maneira diferente frente às variações instrumentais e/ou físico-químicas. O coeficiente linear (a) indica a existência de erro sistemático ao se utilizar a padronização interna, fato que somente é confirmado quando o valor obtido para o coeficiente linear for estatisticamente diferente de zero ${ }^{6}$.

\section{A otimização das condições experimentais}

A otimização do programa de aquecimento envolveu a obtenção de curvas de temperatura de pirólise e atomização para $\mathrm{Ag}, \mathrm{Bi}, \mathrm{Cd}$ e $\mathrm{Pb}$ na presença de $25 \mu \mathrm{g} \mathrm{NH} \mathrm{N}_{4} \mathrm{HO}_{4}$ e $5 \mu \mathrm{g} \mathrm{Pd}+3 \mu \mathrm{g} \mathrm{Mg}$, que são os modificadores químicos freqüentemente empregados nas determinações monoelementares de $\mathrm{Cd}$ e $\mathrm{Pb}^{19,20}$. As condições compromissadas a serem utilizadas para a determinação simultânea de Cd e $\mathrm{Pb}$ devem ser estabelecidas considerando-se o comportamento eletrotérmico observado para esses elementos. Em geral, a temperatura de pirólise é selecionada considerando-se o elemento mais volátil e a temperatura de atomização, o elemento menos volátil ${ }^{12}$. Desta forma, $600 / 1700{ }^{\circ} \mathrm{C}$ e $600 / 1600{ }^{\circ} \mathrm{C}$ foram escolhidas como temperaturas de pirólise e atomização para $25 \mu \mathrm{g} \mathrm{NH}_{4} \mathrm{H}_{2} \mathrm{PO}_{4}$ e $5 \mu \mathrm{g} \mathrm{Pd}+$ $3 \mu \mathrm{g} \mathrm{Mg}$, respectivamente.
A eficiência de um elemento como PI para $\mathrm{Cd}$ e $\mathrm{Pb}$ deve ser considerada sob as melhores condições compromissadas estabelecidas para os analitos. Para isso, 20 medidas consecutivas foram obtidas para $\mathrm{Cd}, \mathrm{Pb}$ e cada um dos elementos testados como PI (Ag e Bi) na presença dos modificadores químicos selecionados. Os parâmetros da regressão linear, calculados a partir dos gráficos de correlação, e o desvio padrão relativo sem e com a padronização interna obtidos

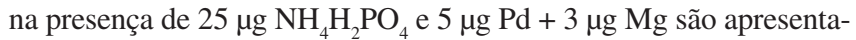
dos nas Tabelas 2 e 3 , respectivamente.

\section{Ag como padrão interno para $\mathrm{Cd}$ e $\mathbf{P b}$}

Os resultados observados quando Ag foi testada como PI indicaram que seu desempenho foi significativamente afetado pelo modificador químico selecionado (Tabelas 2 e 3). Os parâmetros obtidos para a regressão linear dos gráficos entre $\mathrm{Cd} / \mathrm{Ag}$ e $\mathrm{Pb} / \mathrm{Ag}$ mostraram que não há correlação entre os sinais de absorbância desses elementos quando $\mathrm{Pd}+\mathrm{Mg}$ foi utilizado como modificador químico $(\mathrm{Cd} / \mathrm{Ag}: \mathrm{r}=-0,08 \pm 0,03 ; \mathrm{Pb} / \mathrm{Ag}: \mathrm{r}=-0,04 \pm 0,03)$. Por outro lado, os resultados observados na presença de $\mathrm{NH}_{4} \mathrm{H}_{2} \mathrm{PO}_{4}$ indicaram a existência de uma elevada correlação entre os sinais de $\mathrm{Ag} \mathrm{e} \mathrm{Pb}$ $(\mathrm{Cd} / \mathrm{Ag}: \mathrm{r}=0,53 \pm 0,09 ; \mathrm{Pb} / \mathrm{Ag}: \mathrm{r}=0,96 \pm 0,03)$. A influência do modificador químico no desempenho da Ag como PI fica mais evidente quando os gráficos de correlação obtidos na presença de $\mathrm{Pd}+$ $\mathrm{Mg}$ (Figura 1) e $\mathrm{NH}_{4} \mathrm{H}_{2} \mathrm{PO}_{4}$ (Figura 2) são comparados.

Os perfis dos sinais obtidos simultaneamente para $\mathrm{Cd}, \mathrm{Pb}$ e $\mathrm{Ag}$ mostraram que houve uma semelhança temporal na formação dos picos de absorbância quando $\mathrm{NH}_{4} \mathrm{H}_{2} \mathrm{PO}_{4}$ foi utilizado como modificador químico (Figura 3). Nessa situação, o tempo de aparecimento dos sinais de absorbância foi entre 0,62 e 0,91 s (Figura 3) e $0,8 \mathrm{~s}$ foi o tempo necessário para a completa formação de todos os picos $(\mathrm{Cd}=0,60 \mathrm{~s}, \mathrm{~Pb}=0,77 \mathrm{~s}, \mathrm{Ag}=0,62 \mathrm{~s})$. Já na presença de $\mathrm{Pd}+$ $\mathrm{Mg}$, o tempo de aparecimento dos sinais de absorbância variou entre 0,72 e 1,22 s (Figura 4), sendo que o tempo para a completa formação dos picos foi de $0,98,2,93$ e $1,15 \mathrm{~s}$ para $\mathrm{Cd}, \mathrm{Pb}$ e $\mathrm{Ag}$, respectivamente. A maior semelhança temporal dos sinais de absorbância na presença de $\mathrm{NH}_{4} \mathrm{H}_{2} \mathrm{PO}_{4}$ indica que a atomização de $\mathrm{Cd}, \mathrm{Pb}$ e $\mathrm{Ag}$ acontece quase simultaneamente, possibilitando a correção de fenômenos que possam perturbar os eventos que ocorrem durante a etapa de atomização, tais como flutuações de parâmetros instrumentais e interferências na fase vapor.

Tabela 2. Parâmetros obtidos a partir da regressão linear dos gráficos de correlação e desvio padrão relativo (RSD) sem e com a padronização

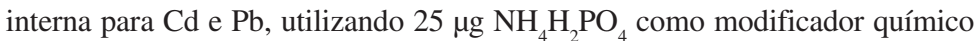

\begin{tabular}{|c|c|c|c|c|c|c|}
\hline & \multirow{2}{*}{$\begin{array}{l}\text { Padrão } \\
\text { Interno }\end{array}$} & \multicolumn{3}{|c|}{ Parâmetros da regressão linear } & \multicolumn{2}{|c|}{$\operatorname{RSD}(\%), \mathrm{n}=20$} \\
\hline & & a & $\mathrm{b}$ & $\mathrm{r}$ & Sem PI & Com PI \\
\hline \multirow[t]{2}{*}{$\mathrm{Cd}$} & $\mathrm{Ag}$ & $-0,97 \pm 0,69$ & $1,94 \pm 0,72$ & $0,53 \pm 0,09$ & 3,1 & 15 \\
\hline & $\mathrm{Bi}$ & $1,29 \pm 0,44$ & $-0,23 \pm 0,46$ & $-0,12 \pm 0,04$ & 2,3 & 4,9 \\
\hline \multirow[t]{2}{*}{$\mathrm{Pb}$} & $\mathrm{Ag}$ & $0,25 \pm 0,04$ & $0,72 \pm 0,05$ & $0,96 \pm 0,03$ & 17 & 7,5 \\
\hline & $\mathrm{Bi}$ & $-0,49 \pm 0,42$ & $1,55 \pm 0,41$ & $0,66 \pm 0,03$ & 1,8 & 3,1 \\
\hline
\end{tabular}

Tabela 3. Parâmetros obtidos a partir da regressão linear dos gráficos de correlação e desvio padrão relativo (RSD) sem e com a padronização interna para $\mathrm{Cd}$ e $\mathrm{Pb}$, utilizando $5 \mu \mathrm{g} \mathrm{Pd}+3 \mu \mathrm{g} \mathrm{Mg}$ como modificador químico

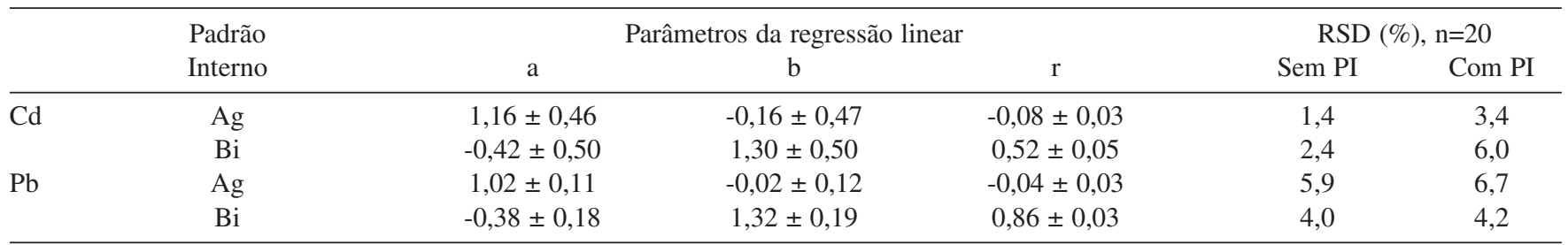



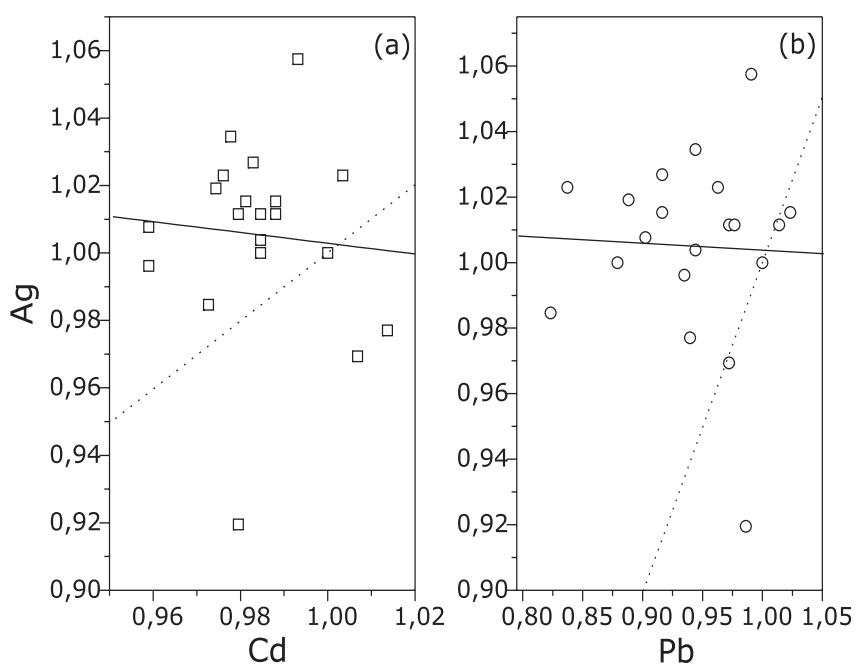

Figura 1. Gráficos de correlação para (a) Ag/Cd e (b) Ag/Pb em meio aquoso, utilizando $5 \mu \mathrm{g} P d+3 \mu \mathrm{g} \mathrm{Mg}$ como modificador químico. (A linha tracejada indica a correlação ideal)

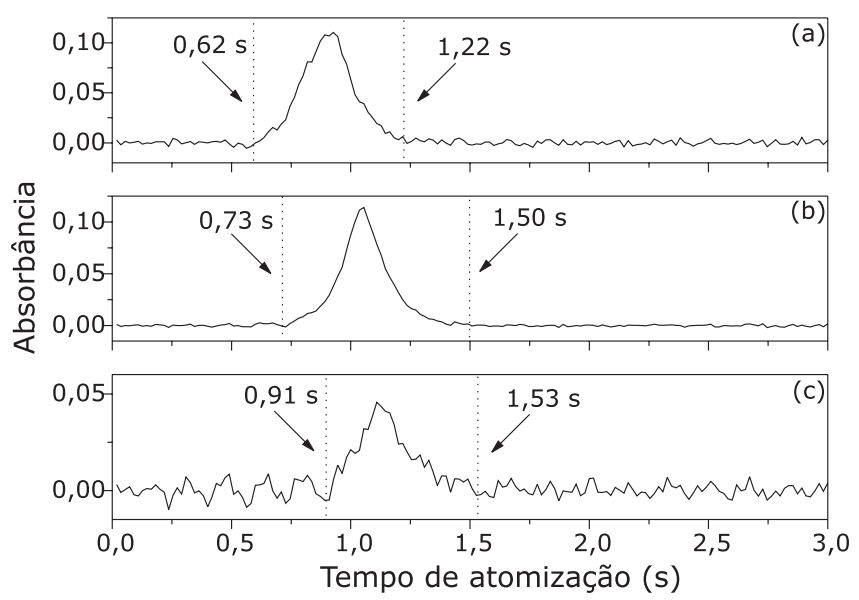

Figura 3. Perfis dos sinais de absorbâncias obtidos para (a) $10 \mathrm{pg} \mathrm{Cd}^{2+}$, (b) $100 \mathrm{pg} \mathrm{Pb}{ }^{2+}$ e (c) $20 \mathrm{pg} \mathrm{Ag}^{+}$em meio aquoso, utilizando $25 \mathrm{\mu g} \mathrm{NH}_{4} \mathrm{H}_{2} \mathrm{PO}_{4}$

\section{Bi como padrão interno para $\mathrm{Cd}$ e $\mathbf{P b}$}

O desempenho apresentado pelo $\mathrm{Bi}$ como PI para $\mathrm{Cd}$ e $\mathrm{Pb}$ foi pior que o esperado, visto que ele é utilizado com sucesso na determinação monoelementar de $\mathrm{Pb}$ em amostras clínicas ${ }^{13}$ e vinho ${ }^{14}$. A avaliação dos resultados obtidos permitiu verificar que a utilização de Bi como PI pode ser afetada significativamente por dois fatores: modificador químico e temperatura de pirólise.

Os parâmetros obtidos a partir da regressão linear dos gráficos de correlação para $\mathrm{Cd} / \mathrm{Bi}$ e $\mathrm{Pb} / \mathrm{Bi}$ na presença de $\mathrm{Pd}+\mathrm{Mg}$ foram melhores que os valores observados para $\mathrm{NH}_{4} \mathrm{H}_{2} \mathrm{PO}_{4}$, em contraste com o que foi verificado para $\mathrm{Ag}$ (Tabelas 2 e 3 ): os coeficientes de correlação obtidos na presença da mistura $\mathrm{Pd}+\mathrm{Mg}(\mathrm{Cd} / \mathrm{Bi}: \mathrm{r}=0,52$ $\pm 0,05, \mathrm{~Pb} / \mathrm{Bi}: \mathrm{r}=0,86 \pm 0,03$ ) foram mais próximos da condição ideal do que os valores observados quando $\mathrm{NH}_{4} \mathrm{H}_{2} \mathrm{PO}_{4}$ foi utilizado como modificador químico $(\mathrm{Cd} / \mathrm{Bi}: \mathrm{r}=-0,12 \pm 0,04, \mathrm{~Pb} / \mathrm{Bi}: \mathrm{r}=0,66$ $\pm 0,03)$.

A eficiência do Bi como PI nas determinações monoelementares de $\mathrm{Pb}$ por ET AAS ${ }^{13,14}$ não foi observada a partir dos dados experimentais obtidos na presença da mistura Pd $+\mathrm{Mg}$ (Tabela 3). O des-
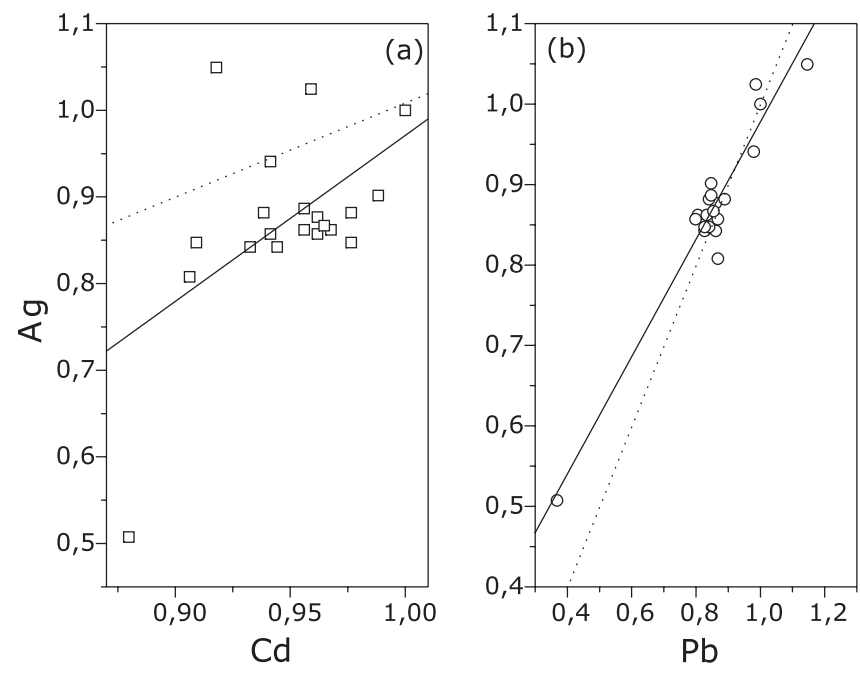

Figura 2. Gráficos de correlação para (a) Ag/Cd e (b) Ag/Pb em meio aquoso, utilizando $25 \mu \mathrm{g} \mathrm{NH}{ }_{4} \mathrm{H}_{2} \mathrm{PO}_{4}$ como modificador químico. (A linha tracejada indica a correlação ideal)

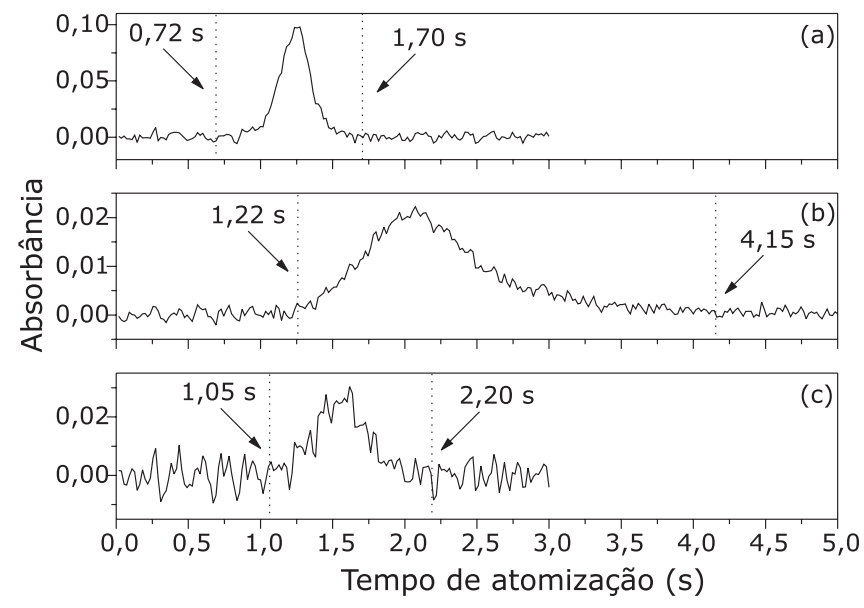

Figura 4. Perfis dos sinais de absorbâncias obtidos para (a) $10 \mathrm{pg} \mathrm{Cd}^{2+}$, (b) $100 \mathrm{pg} \mathrm{Pb}^{2+}$ e (c) $20 \mathrm{pg} \mathrm{Ag}^{+}$em meio aquoso, utilizando $5 \mu \mathrm{g} \mathrm{Pd}+3 \mu \mathrm{g} \mathrm{Mg}$

vio padrão relativo para $\mathrm{Pb}$ ficou praticamente inalterado após a padronização interna com Bi (4,0\% sem correção e 4,2\% com correção), contrastando com a significativa melhoria da precisão dos resultados para a determinação monoelementar de $\mathrm{Pb}^{13,14}$. A comparação das condições experimentais empregadas nessas duas situações permitiu verificar que a temperatura de pirólise foi o parâmetro que sofreu a alteração mais significativa, sendo reduzida de $1000^{\circ} \mathrm{C}$ para $600{ }^{\circ} \mathrm{C}$, a fim de viabilizar a determinação simultânea de $\mathrm{Cd}$ e $\mathrm{Pb}$. Um estudo foi realizado para avaliar qual a influência da temperatura de pirólise no desempenho do Bi como PI: 20 medidas consecutivas foram obtidas para $\mathrm{Cd}, \mathrm{Pb}$ e $\mathrm{Bi}$, na presença de $5 \mu \mathrm{g} \mathrm{Pd}+3 \mu \mathrm{g}$ $\mathrm{Mg}$, selecionando 600 e $1100{ }^{\circ} \mathrm{C}$ como temperaturas de pirólise (Tabela 4).

A repetibilidade dos sinais de Bi não foi alterada com a mudança da temperatura de pirólise, sendo pouco superior a 7\% (Tabela 4). O desvio padrão relativo dos sinais de $\mathrm{Cd}$ aumentou de 2,4 para $6,0 \%$ após a correção com Bi, indicando a baixa eficiência do Bi como PI quando a temperatura de pirólise é baixa $\left(600{ }^{\circ} \mathrm{C}\right)$. De maneira análoga, a precisão dos sinais de $\mathrm{Pb}$ também não foi alterada nessas condições, observando-se um desvio padrão relativo da ordem de 
Tabela 4. Comparação da repetibilidade dos resultados obtidos para $\mathrm{Bi}, \mathrm{Cd}$ e $\mathrm{Pb}$ em meio aquoso, utilizando $5 \mu \mathrm{g} \mathrm{Pd}+3 \mu \mathrm{g} \mathrm{Mg}$ como modificador químico

\begin{tabular}{lccccc}
\hline $\mathrm{Tp}\left({ }^{\circ} \mathrm{C}\right)$ & \multicolumn{5}{c}{$\mathrm{RSD}(\%), \mathrm{n}=20$} \\
& $\mathrm{Bi}$ & $\mathrm{Cd}$ & $\mathrm{Pb}$ & $\mathrm{Cd} / \mathrm{Bi}$ & $\mathrm{Pb} / \mathrm{Bi}$ \\
\hline 600 & 7,4 & 2,4 & 4,0 & 6,0 & 4,1 \\
1100 & 7,3 & $*$ & 7,0 & $*$ & 1,8 \\
\hline
\end{tabular}

* Não foi possível detectar Cd utilizando essa temperatura de pirólise

4\% sem e com a padronização interna com Bi (Tabela 4). Por outro lado, a elevação da temperatura de pirólise para $1100{ }^{\circ} \mathrm{C}$ confirmou que $\mathrm{Bi}$ pode ser utilizado como $\mathrm{PI}$ para $\mathrm{Pb}$. O desvio padrão relativo para os sinais de $\mathrm{Pb}$ foi reduzido de 7,0 para $1,8 \%$ após a correção com Bi (Tabela 4). Essas observações confirmam que, além do modificador químico, a temperatura de pirólise é um parâmetro que pode afetar o desempenho de um elemento como PI.

Uma possível explicação para essa mudança de comportamento pode ser a alteração dos mecanismos de atomização causada pela mudança da temperatura de pirólise. Além de favorecer a eliminação de concomitantes, as espécies químicas precursoras dos átomos livres são formadas durante essa etapa do programa de aquecimento, por meio de processos químicos que dependem da temperatura. A redução da temperatura para $600{ }^{\circ} \mathrm{C}$ alterou o processo de formação dos precursores atômicos e os mecanismos de atomização para Bi e $\mathrm{Pb}$. Verifica-se que há diferentes mecanismos que podem levar à formação de átomos livres de $\mathrm{Bi}$ e $\mathrm{Pb}$, dependendo da temperatura de pirólise e dos precursores atômicos que foram formados. Pode-se supor que os processos físico-químicos e os mecanismos de atomização para $\mathrm{Bi}$ e $\mathrm{Pb}$ são mais parecidos quando a etapa de pirólise ocorre a $1100{ }^{\circ} \mathrm{C}$, permitindo a eficiente correção dos resultados obtidos por meio da padronização interna (Tabela 4). Desta forma, o desempenho do Bi como PI foi comprometido sob condições compromissadas de análise (Tabela 3). Os gráficos de correlação obtidos para $\mathrm{Pb} / \mathrm{Bi}$, utilizando 600 e $1100{ }^{\circ} \mathrm{C}$ durante a etapa de pirólise, confirmam que o $\mathrm{Bi}$ somente é eficiente quando uma elevada temperatura de pirólise é adotada (Figura 5).
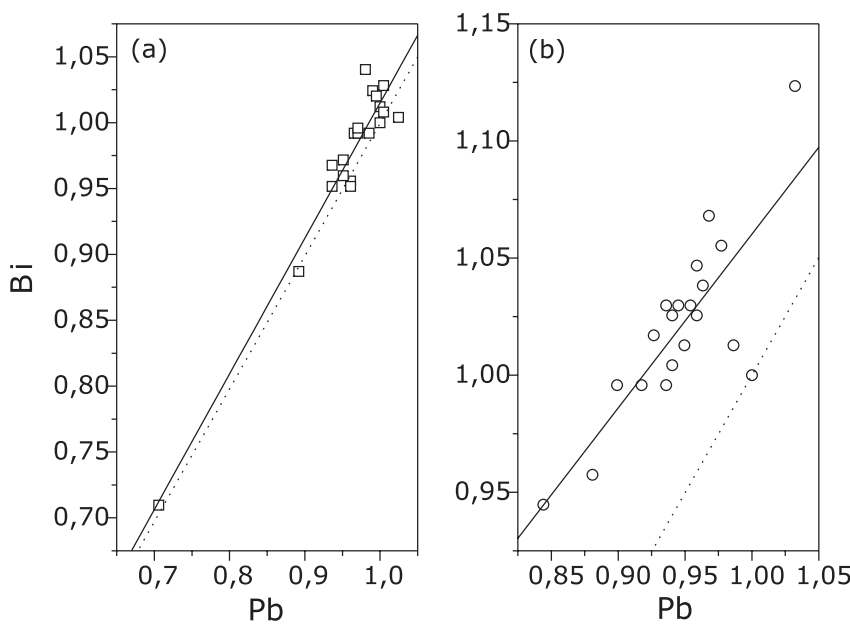

Figura 5. Gráficos de correlação para Bi e Pb em meio aquoso, utilizando $5 \mu \mathrm{g} P d+3 \mu \mathrm{g} \mathrm{Mg}$ como modificador químico e empregando (a) $1100^{\circ} \mathrm{Ce}$ (b) $600^{\circ} \mathrm{C}$ durante a etapa de pirólise. (A linha tracejada indica a correlação ideal)

\section{CONCLUSÕES}

A utilização dos gráficos de correlação nos estudos envolvendo padronização interna em ET AAS auxilia no processo de seleção do PI mais adequado. A necessidade de comparar o desempenho de diferentes elementos testados como PI e as informações valiosas que podem ser extraídas a partir dos gráficos de correlação tornam necessária a utilização desse procedimento.

Considerando os resultados obtidos a partir dos gráficos de correlação, a variação do desvio padrão relativo dos sinais de absorbância sem e com a padronização interna e a avaliação dos tempos de aparecimento e formação dos sinais transientes de absorbância, concluise que a utilização de $\mathrm{Ag}$ como $\mathrm{PI}$ é possível, desde que $\mathrm{NH}_{4} \mathrm{H}_{2} \mathrm{PO}_{4}$ seja o modificador químico selecionado para a determinação simultânea de $\mathrm{Cd}$ e $\mathrm{Pb}$. Com relação ao $\mathrm{Bi}$, conclui-se que a utilização de Bi como PI não é viável sob as condições compromissadas estabelecidas para a determinação simultânea de $\mathrm{Cd}$ e $\mathrm{Pb}$ na presença de $\mathrm{Pd}+\mathrm{Mg}\left(\mathrm{Tp}=600{ }^{\circ} \mathrm{C}, \mathrm{Ta}=1700{ }^{\circ} \mathrm{C}\right)$.

Os testes de repetibilidade que permitiram obter os gráficos de correlação devem ser também realizados na presença da amostra a ser analisada, uma vez que os efeitos interferentes causados pelos concomitantes são intensos em ET AAS e devem ser levados em conta durante o processo de seleção de PI.

\section{AGRADECIMENTOS}

Os autores agradecem ao Conselho Nacional de Desenvolvimento Científico e Tecnológico pelo apoio financeiro. P. R. M. Correia agradece à Fundação de Amparo à Pesquisa do Estado de São Paulo pela bolsa concedida (FAPESP 01/02590-2).

\section{REFERÊNCIAS}

1. Settle, F. A., ed.; Handbook of Instrumental Techniques for Analytical Chemistry, Prentice Hall PTR: Upper Saddle River, 1997, p. 73-80.

2. Lobinski, R.; Marczenko, Z.; Comprehensive Analytical Chemistry: Spectrochemical Trace Analysis for Metals and Metalloids, Elsevier: Amsterdã, 1996, vol. XXX, p. 105-108.

3. Barnett, W. B.; Fassel, V. A.; Kniseley, R. N.; Spectrochim. Acta 1970, 25B, 139

4. Vanhaecke, F.; Vanhoe, H.; Dams, R.; Vandecasteele, C.; Talanta 1992, 39, 737.

5. Kucharkowski, R.; Jankova, D.; Herrmann, E.; John, A.; Fresenius J. Anal. Chem. 1998, 361, 532.

6. Mermet, J. M.; Ivaldi, J. C.; J. Anal. At. Spectrom. 1993, 8, 795.

7. Ivaldi, J. C.; Tyson, J. F.; Spectrochim. Acta 1996, 51B, 1443.

8. Al-Ammar, A. S.; Barnes, R. M.; Spectrochim. Acta 1998, 53B, 1583.

9. Takada, T.; Nakano, K.; Anal. Chim. Acta 1979, 107, 129.

10. Welz, B.; Spectrochim. Acta 1999, 54B, 2081.

11. Erickson, B. E.; Anal. Chem. 2000, 72, 543A.

12. Correia, P. R. M.; Nomura, C. S.; Oliveira, P. V.; Analytica 2003, 5, 52.

13. Radziuk, B.; Romanova, N. P.; Thomassen, Y.; Anal. Commun. 1999, 36, 13.

14. Fernandes, K. G.; Moraes, M.; Gomes Neto, J. A.; Nóbrega, J. A.; Oliveira, P. V.; Analyst 2002, 127, 157.

15. Fernandes, K. G.; Moraes, M.; Gomes Neto, J. A.; Nóbrega, J. A.; Oliveira, P. V.; Quim. Nova 2003, 26, 249.

16. Correia, P. R. M.; Oliveira, P. V.; Gomes Neto, J. A.; Nóbrega, J. A.; J. Anal. At. Spectrom. 2004, 19, 917.

17. Slavin, W.; Manning, D. C.; Carnrick, G. R.; At. Spectros. 1981, 2, 137.

18. L'vov, B. V.; Spectrochim. Acta 1997, 52B, 1.

19. Welz, B.; Schlemmer, G.; Mudakavi, J. R.; J. Anal. At. Spectrom. 1992, 7 1257.

20. Perkin-Elmer Life and Analytical Sciences; The THGA Graphite Furnace: Techniques and Recommended Conditions, Release 1.2: Üeberlingen, 1995. 